CERN-TH/2002-318

hep-th/0212338

\title{
From Boundaries To Conditions Over Superspace
}

\author{
Zheng Yin \\ Theory Division, CERN \\ CH-1211 Geneva 23, Switzerland \\ Zheng.Yin@cern.ch
}

\begin{abstract}
$N=1$ and 2 superconformal boundary conditions are shown to be the consequence of a boundary on the worldsheet superspace with positive codimension in the anticommuting subspace. In addition to the well-known boundary conditions, I also find two new infinite series of $N=2$ boundary states. Their free field realizations are given. A self-contained development of $2 \mathrm{~d}$ superspace leads to new perspectives on this subject.
\end{abstract}




\section{Introduction}

Recently there has been some reexamination of 2 d superconformal field theory with boundaries [1, 2], elaborating the superconformal boundary conditions frequently used [3, 4] for the study of D-branes [5]. The setting was non-linear $\sigma$-models with $N=1$ or $N=2$ worldsheet superconformal symmetry. The analysis was versed in the language of the target space: fields represent coordinates on the target space or sections of vector bundles over it, and coupling constants encodes its geometric feature. This is appropriate for relating the boundary conditions to the geometry of D-branes [4, [6].

The worldsheet origin of boundary suggests an orthogonal viewpoint. Boundary conditions that have to be imposed reflect the modification made to the original theory to consistently accommodate an abrupt end to the space on which it is defined. A fundamental and universal classification of boundary conditions ought to be made solely in worldsheet language without being tied to a particular action formalism. Boundary conformal field theory starts with a choice of boundary conditions imposed between the left and right moving chiral fields. This is most precisely stated in the boundary state formalism. The only constraint seemed to be consistency with the chiral algebra, so they are classified by their outer automorphism group. Those differing by an inner automorphism are mapped into each by symmetries generated by the chiral currents themselves. For a particular type of actions such as non-linear $\sigma$-model, one can make contact with the target space point of view by substituting the concrete realization of the chiral fields into the abstract boundary conditions.

However, the above procedure still does not contain a derivation of the boundary condition itself as the necessary consequence of a boundary. If one doubts the need for such an understanding, consider the following outer automorphism of the Virasoro algebra:f

$$
L_{m} \rightarrow-L_{-m}
$$

There are two problems with using this automorphism to define a boundary condition, such as

$$
\left.\left(L_{m}+\tilde{L}_{m}\right)|\rangle\right)=0
$$

for a boundary state |\rangle$)$. First, algebraic consistency dictates that this automorphism must also flip the sign of the central charge. This is not permissible because in a CFT the central charge is unlike any other element of the Lie algebra - it is just a number, and it has to be the same for the left and right movers. Another problem, which persists even when

\footnotetext{
*I am grateful to V. Kac for pointing out this automorphism.
} 
the central charge vanishes, is that this boundary condition is not local. On a space-like boundary parameterized by $\sigma \in[0,2 \pi)$, It relates the left and right moving stress tensors by

$$
T(\sigma)+\tilde{T}(-\sigma)
$$

As we shall see in $\S 2$, this does not follow from the presence of a boundary.

We can eliminate this problematic automorphism by considering only those leaving the central charge invariant and further require that they have an appropriate local origin in terms of the stress tensor. This makes clear that a derivation of boundary conditions from worldsheet geometry would be desirable. Unfortunately, it is not clear how this could be done for general chiral algebras. Unlike the Virasoro algebra, they seem to represent "internal" degree of freedom mostly independent of the geometry of the worldsheet. However, as will be shown in the paper, boundary conditions for $N=1$ and $N=2$ superconformal algebra (SCA) do have purely worldsheet interpretation, if one is willing to complement the ordinary worldsheet (the even part) with an odd part parameterized by Grassmann "coordinates."

Central to this paper is therefore the use of superspace to describe the action of superconformal symmetries. Worldsheet superspace has long been used in string theory [7, 8], often for writing down Lagrangians with manifest rigid supersymmetry in terms of superfields [9, 10]. Due to the different motivation and purpose of the present work, $2 \mathrm{~d} N=1$ and 2 superspace is developed ab initio in this paper without reference to any Lagrangian or fundamental field. As a consequence issues such as auxiliary fields and off-shell closure is not relevant. Here the Grassmann coordinates are introduced solely as organizational labels for the representations of the singular operator production expansion (sOPE) algebra of the superconformal currents. How they transform under superconformal symmetry is then inferred from the algebra, i.e. superspace emerges as a derived construct. This philosophy differs significantly from that underlying previous works that aimed to combine superspace and superconformal symmetry, where the starting point had been the definition of a superdifferential. For $N=1$ case, the resulting superspace agrees with [1]. For $N=2$ the present results look superficially different from [12, 13, 14, 15]. Both the compact and noncompact versions of $N=2$ SCA make appearance here, classified and differentiated by nonequivalent reality conditions of the superspace. This has not been noted before, but after a field redefinition the compact case does agree with those earlier results on the transformation properties of superconformal tensors.

The technical advantage of this superspace approach is that it allows a systematic and geometric derivation for the boundary conditions. The analysis of pure conformal symmetry can be applied to the superconformal symmetries after the appropriate adaptation. At its 
most essential, a boundary occupies an one dimensional curve on the even part of the superworldsheet. Those conformal symmetries that perturb it in the transverse direction are broken [16]. If the boundary also occupies the whole odd part of the superworldsheet, all supersymmetries are broken. The more interesting case is when the boundary has finite codimension in the odd part as well. It shall be shown in this work that such superboundary can be consistently defined and classified geometrically. They preserve half of the worldsheet supersymmetries. The corresponding boundary conditions for the SCA can also be inferred from geometry, modulo central terms. The latter can then be fixed easily by algebraic arguments. For $N=0$ and 1 the standard boundary conditions are recovered. For $N=2$ $\mathrm{SCA}$, in addition to the well-known $\mathrm{A}$ and $\mathrm{B}$ types of boundary conditions I also find two infinite classes of new ones. They appear for closed boundaries (e.g. boundary state or one-loop diagram of open string) and have to do with the topology of the superworldsheet.

Although this paper is concerned with boundary on the string worldsheet and more generally $2 \mathrm{~d}$ (super)conformal field theory, the idea of using superspace geometry to to classify and derive boundary conditions can be applied to other cases. Boundaries and spacetime defects in supergravity theory and the target space of superstring have recently become an area of intensive research. It would be interesting to see whether the types of analysis presented here would be applicable to them.

The paper is organized as follows. In section 1,the boundary condition of Virasoro algebra is derived from the boundary geometry as a warm-up for the superconformal case. In section $2 N=1$ superconformal symmetry is considered. In section 3 I proceed to $N=2$ superconformal symmetry and find novel boundary states arising from nontrivial superworldsheet topology. In section 4 the novel boundary states are realized in a free theory. While this paper is worldsheet oriented, these concrete solutions can be related to and used for D-branes in $N=2$ compactification [4] and $N=2$ string theories 17, 18.

\section{Boundary Conditions for $N=0$}

What follows can be thought as an elaboration of the argument in section 1 of [16]. It is intended as a warm up drill in which the essential steps needed later for the superconformal cases are run through in a context with little subtlety.

Worldsheet The $2 \mathrm{~d}$ worldsheet is parametrized by coordinates $z$ and $\tilde{z}$. The field theory has conformal symmetry, realized projectively on the Hilbert space of the theory because of 
the conformal anomaly. Denote as usual $T$ and $\tilde{T}$ the decoupled left and right moving stress tensor. The Lie algebra of conformal symmetry can be inferred from their sOPE (eq. 5.9). The operators of the theory are classified into highest weight representations of $\mathrm{T}$, each of which is characterized by a conformal weight $h$ and a chiral primary field $\mathrm{V}$ satisfying the following sOPE:

$$
T(z) \ldots V(w)=\frac{h V(w)}{(z-w)^{2}}+\frac{\partial V(w)}{z-w} .
$$

Infinitesimal conformal transformations are parameterized by two functions $\epsilon(z) \tilde{\epsilon}(\tilde{z})$ and the corresponding charge is f $^{-}$

$$
\frac{1}{2 \pi \imath}(\oint d z \epsilon(z) T(z)+\oint d \tilde{z} \tilde{\epsilon}(\tilde{z}) \tilde{T}(\tilde{z}))
$$

Therefore a chiral primary field $V$ with conformal weight $h$ with respect to $T$ varies as

$$
\delta V=\imath(\epsilon \partial V+h \partial \epsilon V)
$$

Recall that a tensor field $\mathrm{V}$ on a manifold, which may consist of several components, is defined with respect to a group acting on that manifold. It transform generically under an infinitesimal group element $\epsilon$ by

$$
\delta_{\epsilon} \mathrm{V}=\delta_{\epsilon} X^{\mu} \frac{\partial}{\partial X^{\mu}} \mathrm{V}+\Lambda_{\epsilon}^{[\Delta]} \mathrm{V}
$$

Here $\delta_{\epsilon}$ alone represents the action of the group on the coordinate of the manifold and $\Lambda_{\epsilon}^{\Delta}$ represent action of the group on the tensor. $\Delta$ denote properties that distinguish different tensor fields. Hence a chiral primary field of weight $h$ with respect to $T$ is a tensor field for conformal transformation with

$$
\delta_{\epsilon} z=\imath \epsilon(z), \quad \Lambda_{\epsilon}^{[h]}=\imath h \partial \epsilon(z)
$$

Therefore we recovers the spacetime action of a conformal transformation

$$
\delta z=\imath \epsilon, \quad \delta \tilde{z}=\imath \tilde{\epsilon}
$$

Usually conformal transformation is simply defined as such. The procedure here of deriving it from the sOPE of between currents and tensor fields may seem circuitous, but it guarantees the correct result once an SOPE algebra is given. It is well suited for the superconformal cases where geometric intuition is elusive.

\footnotetext{
* The convention for contour integration is counterclockwise for $z$ and clockwise for $\tilde{z}$.
} 
Compatible reparameterization Given (eq. 2.6), one can ask what kind of coordinate redefinition

$$
z^{\prime}=f(z, \tilde{z}), \quad \tilde{z}^{\prime}=f^{\prime}(z, \tilde{z}) .
$$

leaves the form of the variation invariant, i.e.

$$
\delta z^{\prime}=\epsilon^{\prime}(z), \delta z^{\prime}=\tilde{\epsilon}^{\prime}(\tilde{z})
$$

for some $\epsilon^{\prime}$. This gives an automorphism of the sOPE algebra realizable as a reparameterization of the worldsheet. They are important for the classification of boundary conditions and, in conjunction with reality condition, superconformal field theories. The solution for $f$ is clearly that $f$ should only depend on $z$, and $f$ on $\tilde{z}$. Hence they are "almost" conformal transformations themselves. To remove the qualifier "almost" we have to take into account the reality condition.

Reality condition Up to this point I have not really specified the signature of the worldsheet metric. The previous equations are signature independent. The effect of the latter shows up in the reality condition. If the worldsheet is Euclidean, $z$ and $\tilde{z}$ are complex and related by

$$
z^{*}=\tilde{z}
$$

For a Lorentzian worldsheet, $z$ and $\tilde{z}$ are both real and unrelated. For studying field theory in the operator formalism it is necessary that the worldsheet is Lorentzian, and henceforth in this note only this case will be considered.

Generally the reality condition on a Lorentzian worldsheet is $z^{*}=g(z)$ Under reparameterization $z^{\prime}=f(z), g$ transforms as

$$
g(z) \rightarrow g^{\prime}\left(z^{\prime}\right)=f^{*} \circ g \circ f^{(-1)}\left(z^{\prime}\right)
$$

where $f^{(-1)}$ denote the inverse function of $f$. In the literature, one often uses the exponentiated and Wick-rotated notation in which $t$ is replaced by $\tau=\imath t$ and the coordinate $z=\exp \left(\imath \sigma_{L}\right)=\exp (\tau+\imath \sigma)$, with $\sigma$ the compactified spatial direction of the worldsheet. The cylindrical worldsheet is mapped into a two punctured complex plane compactified at infinity. To avoid confusion I shall continue to use $\tilde{z}$ to denote the right moving coordinate and the superscript ${ }^{*}$ to denote conjugation in the Lorentzian sense with the appropriate analytic continuation. The reality condition in this convention becomes

$$
z^{*}=1 / z
$$


Unless specified otherwise, I shall follow this convention in the rest of this work. This is especially convenient for performing contour integration with the sOPE's but introduces some complication for the boundary and reality conditions.

Conformal transformation should of course respect the reality condition, which leads to the reality conditions for $\epsilon$ and $\tilde{\epsilon}$ :

$$
(\epsilon(z))^{*}=z^{-2} \epsilon(z), \quad(\tilde{\epsilon}(\tilde{z}))^{*}=\tilde{z}^{-2} \tilde{\epsilon}(\tilde{z})
$$

The reparameterization consider earlier should also leave the reality condition invariant. This leads to the reality condition on $f$. It still best to state it in terms of the unexponentiated, un-Wick-rotated coordinates: $f\left(\sigma_{L}\right)$ and $\tilde{f}\left(\sigma_{R}\right)$ are both real. In this paper, reparameterizations compatible with infinitesimal (super)conformal transformations and a chosen reality condition are called reparameterization automorphisms. They obviously form a group. They are important for classifying boundary conditions. It is also clear that transition functions between different patches of the (super)worldsheet must be elements of this group. Note that in the present case they are just the usual conformal transformations. They are generated by (eq. 2.2) subjected to the appropriate reality conditions on $\epsilon$ plus the Hermiticity of $T$ and $\tilde{T}$. It means that they are in fact inner automorphisms. For the superconformal cases this will no longer be true.

Boundary condition Now consider a boundary on the Lorentzian worldsheet. Locally the boundary can be any one of three types: time-like, space-like, and null. The last type is located at a fixed value of say $z$ but have no restriction locally for $\tilde{z}$. It leads to intriguing pathologies and is rarely used in the literature. Henceforth I shall require the boundary to be nowhere null. Thus they are globally either time-like or space-like. Analytically this can be seen as follows. A boundary condition that is nowhere null can be written as

$$
\tilde{z}=k(z) .
$$

Then a reparameterization automorphism acts on $k$ by

$$
k \rightarrow k^{\prime}=\tilde{f} \circ k \circ f^{(-1)}
$$

Equivalence classes of $k$ with respect to this classifies the boundary condition. A time-like boundary can always be brought to be the positive real axis.

$$
\tilde{z}=z \geq 0
$$


and space-like boundary to

$$
z \tilde{z}=1 .
$$

The conformal transformations leaving these equation invariant are respectively

$$
\epsilon=\left.\tilde{\epsilon}\right|^{z=\bar{z}}
$$

and

$$
\bar{z} \epsilon=-\left.z \tilde{\epsilon}\right|^{|z|=1}
$$

What does this imply for the currents and charges in the theory that generates these symmetries? Consider first an arbitrary symmetry of the worldsheet that leaves invariant a boundary of the space on which the theory is defined.For a local quantum field theory, features of the space on which it is defined is immune from quantum fluctuation, so a boundary and the symmetries preserving it should manifest themselves up to quantum anomalies. Furthermore, quantum anomaly can be computed locally in the bulk. Therefore a boundary preserving symmetry is realized in the theory if and only if the same symmetry exist in the absence of the boundary, and with the same cocycle if the realization is projective.

The manner in which it is realized depend on the type of the boundary. If the boundary is space-like, it represents a state in the QFT, [1 while if it is time-like, the boundary modifies the theory itself. In the language of string theory, it is a boundary state in the close string theory versus an open string theory in its own right. Suppose the symmetry is generated by some charge $Q$ constructed from some conserved current $j_{\mu}$ as usual by $Q=\int d^{D-1} x J_{0}$. If the boundary is space-like and represented by some boundary state |\rangle$)$, the latter should be invariant under the charge:

$$
Q|\rangle)=0
$$

If the boundary is time-like, the charge $Q$ remains a constant of motion. It follows that the boundary contribution to $\dot{Q}$ vanishes:

$$
\int_{\partial \Sigma} d A \cdot J=0
$$

This is a constraint on the open string theory stipulating that no net flux of $Q$ charge leak through the boundary.

Now apply this to conformal symmetries, where

$$
j_{z}=\epsilon T, \quad j_{\bar{z}}=\tilde{\epsilon} \tilde{T} .
$$

\footnotetext{
${ }^{\dagger}$ It is not a state in the proper Hilbert space because it would generically have infinite norm, but this is not a relevant issue here.
} 
Substituting this with (eq. 2.17) in (eq. 2.2), one finds after factoring out the arbitrary $\epsilon$ that

$$
T-\tilde{T}=\left.0\right|^{\sigma=0}
$$

for the open string theory and

$$
\left.\left(T-\frac{\tilde{z}^{2}}{z^{2}} \tilde{T}\right)|\rangle\right)=0
$$

for the boundary state. Since

$$
T=\sum_{n \in \mathbb{Z}} L_{m} z^{-m-2}, \quad \tilde{T}=\sum_{n \in \mathbb{Z}} \tilde{L}_{m} \tilde{z}^{-m-2} .
$$

the last equation means

$$
\left.\left(L_{m}-\tilde{L}_{-m}\right)|\rangle\right)=0
$$

To be precise, the above procedure gives only the correct boundary condition modulo central terms. It is easy to verify algebraically that that no correction is needed in this case. In fact these are the usual boundary conditions for CFT.

Of course one can think of other boundary conditions. A priori, the only algebraic constraint is that the relation between the left and right moving chiral fields are consistent with their algebra, therefore boundary conditions are corresponds to automorphism of the symmetry algebra that preserves the central charge and appropriate Hermiticity condition. For Virasoro algebra that just leaves the inner automorphisms, those generated by $L_{m}$ themselves and corresponding to conformal transformations. On the other hand, the above analysis has shown that the boundary condition on the stress tensor, following strictly the requirement of preserving the geometric boundary, is (eq. 2.22) or (eq. 2.25) for time-like or space-like boundaries, after the boundary is brought to canonical forms by a conformal transformation. Therefore the other possible boundary conditions just correspond to boundaries that have not been brought to one of the two canonical forms. Had there been an automorphisms that do not have some origin from geometry (e.g. a boundary or a crosscap), their interpretation in boundary CFT would have been suspect.

When a bigger chiral algebra is known for a CFT, one should likewise consider boundary conditions for them as well [19, 16]. However, these symmetries in general acts on "internal" degrees of freedom rather than worldsheet. In the language of (eq. 2.4), they only shows up in $\Lambda_{\epsilon}^{[\Delta]}$. The method developed above relies on $\delta_{\epsilon} X^{\mu}$ and thus cannot be used to give a geometric derivation of their boundary conditions. However, for superconformal symmetries this is possible by enlarging the notion of worldsheet, and for these cases the systematic approach of this work displays its power. It is amusing to note that the very notion of 
superspace will be derived ab initio from the sOPE algebra of the (super)currents rather than conjured out of the void. Even here one may take the view that the worldsheet coordinate $z$ and $\tilde{z}$ was reconstructed from the Virasoro algebra.

\section{Boundary Conditions for $N=1$}

$N=1$ superworldsheet The $N=1$ superconformal algebra is the simplest extension to Virasoro algebra with an interpretation purely in terms of superworldsheet geometry. Consider the left moving part. The $N=1$ superconformal algebra (SCA) complements $T$ with a superpartner $G$ and their sOPE's is given in the appendix. From this one may determine the multiplet structure of irreducible tensor fields and their sOPE's with $T$ and $G$. There is just one kind, again characterized by a conformal weight $h$ but now consists of two components fields. They can be combined into a superfield as $\mathrm{V}=V^{\downarrow}+\theta V^{\uparrow}$ by introducing a Grassmann variable $\theta$. For the time being it is merely an nilpotent label.

$V^{\downarrow}$ and $V^{\uparrow}$ are both chiral primary fields of $T$ with conformal weight $h$ and $h+1 / 2$ respectively. Their sOPE's with $G$ are

$$
\begin{aligned}
& G(z) \ldots V^{\downarrow}(w)=\frac{V^{\uparrow}}{z-w}, \\
& G(z) \ldots V^{\uparrow}(w)=\frac{2 h \partial V^{\downarrow}}{(z-w)^{2}}+\frac{\partial V^{\downarrow}}{z-w}
\end{aligned}
$$

Infinitesimal $N=1$ superconformal transformations are generated by

$$
\frac{1}{2 \pi \imath}(\oint d z \epsilon(z) T(z)+\oint d z a(z) G(z)) .
$$

where $a$ is the Grassmann valued parameters for supersymmetry. By contour integration of the OPE (eq. 3.1), one finds that

$$
\begin{aligned}
\delta \mathrm{V} & =\imath\left[\epsilon \partial+\frac{1}{2} \partial \epsilon \theta \partial_{\theta}+h \partial \epsilon\right] \mathrm{V} \\
& +\imath\left[-a \theta \partial+a \partial_{\theta}-2 h \partial a \theta\right] \mathrm{V}
\end{aligned}
$$

From this I deduce the action of the superconformal transformation on the superspace coordinate:

$$
\delta z=\imath(\epsilon-a \theta), \quad \delta \theta=\imath\left(\frac{1}{2} \partial \epsilon \theta+a\right),
$$

and $\theta$ now emerges as a coordinate on a superworldsheet. One then does the same for the right movers, introducing $\tilde{\theta}$ in the process. The superworldsheet is therefore make up of 
an even part parameterized by $z$ and $\tilde{z}$ and an odd part parameterized by $\theta$ and $\tilde{\theta}$. To be precise, the latter is fibered over the former. The transition function are elements of the reparameterization automorphism discussed below.

Compatible reparameterization Consider the reparameterization

$$
z^{\prime}=f(z), \quad \theta^{\prime}=\mathbf{f}(z) \theta
$$

that leaves (eq. 3.4) invariant. In fact I have already used it to eliminate $\bar{z}$ dependence and mixing with $\tilde{\theta}$. Solving now for

$$
\delta z^{\prime}=\epsilon^{\prime}-a^{\prime} \theta^{\prime}, \quad \delta \theta^{\prime}=\frac{1}{2} \frac{\partial \epsilon^{\prime}}{\partial f}+a^{\prime}
$$

one obtains $\mathbf{f}=\sqrt{\partial f} \hat{\mathbf{f}}$ with

$$
\hat{\mathbf{f}}= \pm 1
$$

Here the $\sqrt{\partial f}$ factor merely reflects that $\theta$ is really a conformal tensor of weight $-1 / 2$.

Reality condition If the $V^{\downarrow}$ is a real field, we expect the other components of the same multiplet is also real in an irreducible multiplet. This necessitates a reality condition on $\theta$. It can only be of the form

$$
\theta^{*}=\mathbf{g}(z) \theta
$$

which also has to be be invariant under (eq. 3.4). This leads to $\epsilon^{*}=z^{-2} \epsilon$ as before and also

$$
a^{*}=-\mathbf{g} a \text {. }
$$

$\mathbf{g}$ has to satisfy $\mathbf{g}=\sqrt{-\partial g} \hat{\mathbf{g}}=\hat{\mathbf{g}} / z$ with: $\hat{\mathbf{g}}= \pm 1$. Reality conditions are equivalent if and only if they are related by a compatible reparameterization $(f, \mathbf{f})$ through

$$
\mathrm{g} \rightarrow \mathbf{f}^{*} \mathbf{g f}^{-1}
$$

It is easy to see that $f(z)=1 / z$ will flip the sign of $\hat{\mathbf{g}}$. Hence there is only one type of reality condition and one can set $\hat{\mathbf{g}}=1$.

It also follows that the full reparameterization automorphism group, $(f, \mathbf{f})$ that leaves $(g, \mathbf{g})$ invariant, consists of conformal transformation plus conformal transformations combined with flipping the sign of odd elements. The two correspond to the two choices of sign for $\hat{\mathbf{f}}$. The ones with $\hat{\mathbf{f}}=-1$ are disconnected from the identity and not generated by $T$. This choice corresponds to an outer automorphism of the $N=1 \mathrm{SCA}$. A transition function for the fiber of odd space over the even space has the same choice. The topological classes they allow are the spin structures on the bosonic worldsheet. The analysis of the $N=1$ SCA for the right movers is exactly the same. 
Boundary condition Now let us add a boundary to the worldsheet superspace parameterized by $z, \theta$ and $\tilde{z}, \tilde{\theta}$. The boundary must have a locus on the even part of the worldsheet. Again, it can be brought into either positive real axis (eq. 2.15) or the unit circle (eq. 2.16). Requiring these equations to be invariant under the superconformal transformations (eq. 3.4) one finds what infinitesimal transformation leaves the boundary invariant. To avoid repetition I will concentrate on the case of space-like boundary at $\tilde{z} z=1$. The other cases are entirely analogous and easily related to this one.

Substituting (eq. 3.4) and its right mover counterparts in (eq. 2.16), one finds the conditions for the infinitesimal parameter on the boundary. In addition to (eq. 2.18), there is also

$$
\tilde{z} a \theta=z \tilde{a} \tilde{\theta} .
$$

If this has to hold for arbitrary $\theta$ and $\tilde{\theta}$, the only solution is $a=\tilde{a}=0$, i.e. this boundary breaks all superconformal transformation. The only boundary condition is (eq. 2.25). However, (eq. 3.11) can have nontrivial solutions for $a$ if we consider a boundary which does not span the whole odd part of the superworldsheet, but instead is localized along a "superline" defined by

$$
\tilde{\theta}=\mathbf{k}(\sigma) \theta .
$$

This is the most general conditions that can be imposed on the odd part of the superworldsheet. (Eq. 3.11) then has the solution

$$
\tilde{z} a+z \mathbf{k} \tilde{a}=0 .
$$

For consistency, one also has to require (eq. 3.12) to be invariant under such supersymmetric transformation. A straightforward calculation leads to the constraint $\mathbf{k}=\imath e^{-\imath \sigma} \hat{\mathbf{k}}$ with

$$
\hat{\mathbf{k}}= \pm 1
$$

Again the $\imath e^{-\imath \sigma}$ phase reflects the tensor nature of $\theta$ and $\tilde{\theta}$. The more important information lies in $\hat{\mathbf{k}}$. At first sight, the two choices of signs seems equivalent. Under a reparameterization automorphism $(f, \mathbf{f})$ that leave fixed the bosonic boundary,

$$
\mathbf{k} \rightarrow \tilde{\mathbf{f}} \mathbf{k f}^{-1}
$$

Hence one can flips the sign of $\hat{\mathbf{k}}$ by $\mathbf{f}=-1$ with trivial $f, \tilde{f}$ and $\tilde{\mathbf{f}}$. However, doing so would upset the boundary conditions else where. Consider a worldsheet with more than one boundaries. It is not possible to flips the sign of $\mathbf{k}$ for one without affecting all the other. This essentially has to do with the above mentioned fact that reparameterization 
automorphisms with $\mathbf{f}=-1$ is disconnected from identity. Therefore these two possibilities for $\mathbf{k}$ correspond to two different types of boundaries.

Corresponding to them, one has

$$
e^{-\sigma / 2} a \pm \imath e^{-\sigma / 2} \tilde{a}=0 .
$$

on the boundary, leading to the equation

$$
\left.G_{m} \pm \imath \tilde{G}_{-m}|\rangle\right)=0
$$

This is the well-known boundary condition for the supercurrent used for D-branes in Type II string theory. The above analysis shows that it corresponds to having a boundary in superspace defined by

$$
e^{\imath \sigma / 2} \tilde{\theta}= \pm \imath e^{-\imath \sigma / 2} \theta
$$

along with the more usual bosonic boundary. It also shows how limited the possibility is. Any other space-like boundary one can define is related to this one by a superconformal transformation. For an annulus worldsheet with two boundaries, the relative choice of signs on each translates to the choice of NS or R type of twisted $N=1$ algebra for open string theory and the possibility of inserting a G-parity operator in the close string/boundary state picture.

\section{Boundary Conditions for $N=2$}

Multiplet structure Compared to $N=1 \mathrm{SCA}, N=2 \mathrm{SCA}$ has two supercurrents $G^{ \pm}$ instead of one, and has an affine $U(1)$ current $J$ in addition. Their sOPE's are given in the appendix. From this and the Jacobi identity it is a straightforward but tedious exercise to show that it has exactly three families of irreducible tensor multiplets. The more generic family is characterized by two numbers, $h$, and $\lambda$ and consists of four fields, which we denote as $V^{\downarrow}, V^{-}, V^{+}, V^{\uparrow}$ respectively. We shall call it the long multiplet. Their sOPE's with the $N=2$ supercurrents are

$$
\begin{aligned}
G^{+}(z) \ldots V^{\downarrow}(w) & =\frac{V^{+}(w)}{z-w}, \\
G^{-}(z) \ldots V^{\downarrow}(w) & =\frac{V^{-}(w)}{z-w}, \\
G^{+}(z) \ldots V^{+}(w) & =G^{-}(z) \ldots V^{-}(w)=0, \\
G^{+}(z) \ldots V^{-}(w) & =\frac{h(1+\lambda) V^{\downarrow}(w)}{(z-w)^{2}}+\frac{\frac{1}{2}(1+\lambda) \partial V^{\downarrow}}{z-w}+\frac{V^{\uparrow}(w)}{z-w},
\end{aligned}
$$




$$
\begin{aligned}
G^{-}(z) \ldots V^{+}(w) & =\frac{h(1-\lambda) V^{\downarrow}(w)}{(z-w)^{2}}+\frac{\frac{1}{2}(1-\lambda) \partial V^{\downarrow}(w)}{z-w}-\frac{V^{\uparrow}(w)}{z-w} \\
G^{+}(z) \ldots V^{\uparrow}(w) & =-\frac{(h+1 / 2)(1+\lambda) V^{+}(w)}{(z-w)^{2}}-\frac{\frac{1}{2}(1+\lambda) \partial V^{+}(w)}{z-w} \\
G^{-}(z) \ldots V^{\uparrow}(w) & =+\frac{(h+1 / 2)(1-\lambda) V^{-}(w)}{(z-w)^{2}}+\frac{\frac{1}{2}(1-\lambda) \partial V^{-}(w)}{z-w}
\end{aligned}
$$

$V^{\downarrow}$, and $V^{ \pm}$are primary with respect to both with (weight, charge) being $(h, 2 h \lambda),(h+$ $1 / 2,2 h \lambda \pm 1$ ) respectively. As for $V^{\uparrow}$, it is primary门 with respect to $T$ with conformal weight $h+1$ but has the following OPE with $J$ :

$$
J(z) \ldots V^{\uparrow}(w)=\frac{h\left(1-\lambda^{2}\right) V^{\downarrow}(w)}{(z-w)^{2}}+\frac{2 h \lambda V^{\uparrow}(w)}{z-w}
$$

Note that $q=2 h \lambda$ is the $U(1)$ charge of $V^{\downarrow}$ and what is often called the charge of the whole multiplet. When $\lambda= \pm 1$ the long multiplet becomes reducible into two shorter types of multiplets. For our purpose the long multiplet is sufficient.

$N=2$ superworldsheet Without further ado let us turn a long multiplet into a superfield

$$
\mathrm{V}=V^{\downarrow}+\theta^{+} V^{-}+\theta^{-} V^{+}+\theta^{+} \theta^{-} V^{\uparrow}
$$

by introducing two Grassmann variables $\theta^{ \pm}$. Now consider an infinitesimal $N=2$ superconformal transformation generated by

$$
\frac{1}{2 \pi \imath}\left(\oint d z \epsilon(z) T(z)+\oint d z \xi(z) J(z)+\oint d z a^{+}(z) G^{-}(z)+\oint d z a^{-}(z) G^{+}(z)\right) .
$$

Here $\epsilon$ and $\xi$ are ordinary $\mathbb{C}$ valued while $a^{ \pm}$are Grassmann valued. By performing contour integration with the above OPE's, one find that

$$
\begin{aligned}
\delta \mathrm{V} & =\imath\left[\epsilon \partial+\frac{1}{2} \partial \epsilon\left(\theta^{+} \partial_{\theta^{+}}+\theta^{-} \partial_{\theta^{-}}\right)+h \partial \epsilon\right] V \\
& +\imath\left[\xi\left(-\theta^{+} \partial_{\theta^{+}}+\theta^{-} \partial_{\theta^{-}}\right)+2 h \lambda \xi+h\left(1-\lambda^{2}\right) \partial \xi \theta^{+} \theta^{-}\right] V
\end{aligned}
$$

${ }^{*} \Phi$ is primary with respect to a affine $U(1)$ current $J$ with charge $q$ if its sOPE with $J$ is

$$
J(z) \ldots \Phi(w)=\frac{q \Phi(w)}{z-w} .
$$

$\dagger$ [14] also contained explicit transformation properties of tensor components. There the top component is not primary with respect to $T$. It is related to the convention here by $V^{\uparrow}+(\lambda / 2) \partial V^{\downarrow}$. 


$$
\begin{aligned}
& +\imath\left[-\frac{1}{2}(1-\lambda) a^{+} \theta^{-} \partial+a^{+} \partial_{\theta^{+}}+(1-\lambda) \partial a^{+}\left(\frac{1}{2} \theta^{+} \theta^{-} \partial_{\theta_{+}}+h \theta^{+}\right)\right] V \\
& +\quad \imath\left[-\frac{1}{2}(1+\lambda) a^{-} \theta^{+} \partial+a^{-} \partial_{\theta^{-}}-(1+\lambda) \partial a^{-}\left(\frac{1}{2} \theta^{+} \theta^{-} \partial_{\theta_{-}}+h \theta^{-}\right)\right] V .
\end{aligned}
$$

A novel aspect of $N=2 \mathrm{SCA}$ is that the derivation part of the variation contain dependence on $\lambda$. One can split it into two pieces as $\lambda=\gamma+\breve{\lambda}$ with some arbitrary number $\gamma$, and treats it as an universal piece for any tensor types. It is clear that it should close by itself in accordance with $N=2$ algebra for arbitrary $\gamma$. Then the action of $N=2$ SCA on superspace is:

$$
\begin{aligned}
\delta z & =\imath\left(\epsilon-\frac{1}{2}\left(a^{+} \theta^{-}+a^{-} \theta^{+}\right)+\frac{\gamma}{2}\left(a^{+} \theta^{-}-a^{-} \theta^{+}\right)\right), \\
\delta \theta^{ \pm} & =\imath\left( \pm \xi \theta^{ \pm}+a^{ \pm} \pm \frac{1}{2} \partial a^{ \pm} \theta^{+} \theta^{-}-\frac{1}{2} \gamma \partial a^{ \pm} \theta^{+} \theta^{-}\right)
\end{aligned}
$$

We can write it more compactly by grouping $\theta^{ \pm}$into a column vector $\theta$ and $a^{ \pm}$into $a$ :

$$
a=\left(\begin{array}{l}
a^{+} \\
a^{-}
\end{array}\right), \quad \theta=\left(\begin{array}{c}
\theta^{+} \\
\theta^{-}
\end{array}\right) .
$$

Then (eq. 4.6) becomes

$$
\begin{aligned}
\delta z & =\imath\left[\epsilon+\frac{\theta^{\top}}{2}\left(\sigma^{1}+\imath \gamma \sigma^{2}\right) a\right] \\
\delta \theta & =\imath\left[\frac{1}{2} \partial \epsilon \theta-\xi \sigma^{3} \theta+a+\frac{1}{2} \theta \theta^{\top}\left(\sigma^{1}+\imath \gamma \sigma^{2}\right) \partial a\right]
\end{aligned}
$$

The $N=2$ superworldsheet has a bigger odd part, parameterized by $\theta^{ \pm}$and $\tilde{\theta}^{ \pm}$. It is fibered over the even part. Note that so far $\gamma$ is an arbitrary parameter labelling $N=2$ superspace. Below it will be shown that different values of $\gamma$ are in fact equivalent and can be set to zero.

Compatible reparameterization Along the same line as before, consider now reparameterization of the superspace coordinate that is compatible with infinitesimal $N=2$ superconformal transformations. Define

$$
z^{\prime}=f(z)+\frac{\omega}{2} \theta^{\top} \imath \sigma^{2} \theta, \quad \theta^{\prime}=\mathbf{f}(z) \theta
$$

where $\theta$ is a complex two-by-two matrix. This is the most general holomorphic reparametrization of superspace with "bosonic" parameters. Requiring

$$
\begin{aligned}
\delta z^{\prime} & =\imath\left[\epsilon^{\prime}+\frac{\partial \epsilon^{\prime}}{\partial f} \frac{\omega}{2} \theta^{\top} \imath \sigma^{2} \theta+\frac{\theta^{\prime \top}}{2}\left(\sigma^{1}+\imath \gamma^{\prime} \sigma^{2}\right) a^{\prime}\right] \\
\delta \theta^{\prime} & =\imath\left[\frac{1}{2} \partial \epsilon^{\prime} \theta^{\prime}-\xi^{\prime} \sigma^{3} \theta^{\prime}+a^{\prime}+\frac{\partial a^{\prime}}{\partial f} \frac{\omega}{2} \theta^{\top} \imath \sigma^{2} \theta+\frac{1}{2} \theta^{\prime} \theta^{\prime \top}\left(\sigma^{1}+\imath \gamma^{\prime} \sigma^{2}\right) \frac{\partial a^{\prime}}{\partial f}\right]
\end{aligned}
$$


leads to the following expression for the transformation of the parameters:

$$
\epsilon^{\prime}=\partial f \epsilon, \quad a^{\prime}=\mathbf{f} a, \quad \xi^{\prime} \sigma^{3}=\xi \hat{\mathbf{f}} \sigma^{3} \hat{\mathbf{f}}^{-1}-\partial \hat{\mathbf{f}} \hat{\mathbf{f}}^{-1} \epsilon
$$

where $\hat{\mathbf{f}} \equiv \mathbf{f} / \sqrt{\partial f}$. There are a number of constraints on $\hat{\mathbf{f}}$ and $\omega$, a minimal set of which are

$$
\partial \hat{\omega}=0, \quad \sigma^{3} \hat{\mathbf{f}} \sigma^{3}= \pm \hat{\mathbf{f}}, \quad \hat{\mathbf{f}}^{\top}\left(\sigma^{1}+\imath \gamma^{\prime} \sigma^{2}\right) \hat{\mathbf{f}}=\left(\sigma^{1}+\imath(\gamma+\hat{\omega}) \sigma^{2}\right)
$$

where $\hat{\omega}=\omega / \partial f$. Corresponding to the choice of sign in the last equation, there are two families of solutions:

$$
\hat{\mathbf{f}}=e^{\phi \sigma^{3}}, \quad \gamma^{\prime}=\gamma+\hat{\omega}
$$

and

$$
\hat{\mathbf{f}}=\sigma^{1} e^{\phi \sigma^{3}}, \quad \gamma^{\prime}=-\gamma-\hat{\omega}
$$

Here $\phi$ is an arbitrary complex number. Using $\hat{\omega}$ we will set $\gamma$ to 0 without loss of generality. This choice fixes the $\omega$ parameter in to 0 as well.

Reality condition To ensure the right number of degrees of freedom, one has to impose

$$
\theta^{*}=\mathbf{g} \theta
$$

where $\mathbf{g}$ is a 2-by-2 matrix satisfying the consistency condition

$$
\mathbf{g}^{*} \mathbf{g}=1
$$

In addition, (eq. 4.15) has to be compatible with the conformal transformation (eq. 4.8). This leads to

$$
a^{*}=-\mathbf{g} a, \quad \xi^{*} \sigma^{3}=-\xi \hat{\mathbf{g}} \sigma^{3} \hat{\mathbf{g}}^{-1}+\epsilon \partial \hat{\beta} \hat{\beta}^{-1}
$$

where $\hat{\mathbf{g}}=\mathbf{g} / \sqrt{-\partial g}$. $\hat{\mathbf{g}}$ satisfies the following consistency conditions:

$$
\hat{\mathbf{g}}^{*} \hat{\mathbf{g}}=1, \quad \sigma^{3} \hat{\mathbf{g}} \sigma^{3}= \pm \hat{\mathbf{g}}, \quad \hat{\mathbf{g}}^{\top} \sigma^{1} \hat{\mathbf{g}}=\sigma^{1}
$$

Corresponding to the choice of sign in the 2nd equation, there are two families of solutions both parameterized by a real number $p$

$$
\hat{\mathbf{g}}=e^{\imath p \sigma^{3}} .
$$

and

$$
\hat{\mathrm{g}}= \pm \sigma^{1} e^{p \sigma^{3}} .
$$


The reality condition again transform under the reparameterization $\mathbf{f}$ as

$$
\mathrm{g} \rightarrow \mathbf{f}^{*} \mathbf{g f}^{-1} .
$$

It is easy to see that one can always set $p$ to 0 (but see the caveat below) and fix the sign in (eq. 4.20). The stabilizers are

$$
\hat{\mathbf{f}}= \pm e^{\phi \sigma^{3}} \text { and } \sigma^{1} \pm e^{\phi \sigma^{3}}
$$

for $\hat{\mathbf{g}}=\mathbb{I}$ and

$$
\hat{\mathbf{f}}=e^{\imath \phi \sigma^{3}} \text { and } \sigma^{1} e^{\imath \phi \sigma^{3}}
$$

for $\hat{\mathbf{g}}= \pm \sigma^{1}$.

The meaning of the reparameterization automorphism is evident. $\mathbf{f}$ is a function of $z$ taking value in a group $\mathcal{G}$. For $\mathbf{g}=1 \mathcal{G}$ is $O(1,1)$ while for $\hat{\mathbf{g}}=\sigma$ it is $O(2)$. Ignore for now the topology of the worldsheet, the group of reparameterization automorphisms has the same number of components as $\mathcal{G} . O(1,1)$ has four while $O(2)$ has two. These components correspond to the various solutions in (eq. 4.22) and (eq. 1.23) and in obvious ways. They are the structure group of the Grassmann fibers. The global aspect of the compact case has been considered in [13].

All things considered, there are two nonequivalent reality conditions. They correspond to different $N=2$ SCA's. For $\hat{\mathrm{g}}=1$, the abelian group generated by $J_{0}$ is noncompact this is known as the noncompact $N=2 \mathrm{SCA}$. It is easy to see that it cannot have unitary highest weight representation. On the other hand, $\mathrm{g}=\sigma^{1}$ gives the conventional compact $N=2$ SCA with an $U(1)$ symmetry. Most of the studies on $N=2$ SCA and SCFT have been on the compact version.

I should note here one caveat in the previous discussion. When, as in string theory, the worldsheet is compact in its spatial direction $\sigma, \hat{\mathrm{g}}$ in (eq. 1.19) can have nontrivial winding as it takes value in $U(1)$. This can still be brought to the canonical form of $\hat{\mathbf{g}}=1$ by using $\hat{\mathbf{f}}$ with half as much winding. However when the winding number of $\hat{\mathrm{g}}$ is odd, the necessary $\hat{\mathbf{f}}$ is antiperiodic around $\sigma$. Its effect is to make $G^{ \pm}$also anti-periodic. This gives a twisted version of the $N=2$ mode algebra with half integer modes for the supercurrents. It is related to the $Z_{2}$ center of $O(1,1)$.

Boundary Now introduce a boundary to the $N=2$ superworldsheet. Again concentrate on the case of $z \bar{z}=1$. Invariance of this expression under the $N=2$ superconformal transformation (eq. 4.6) leads to

$$
z^{-1} \theta^{\top} \sigma^{1} a+\tilde{z}^{-1} \tilde{\theta}^{\top} \sigma^{1} \tilde{a}=0
$$


in addition to (eq. 2.18). If the boundary occupies the whole odd part of the superworldsheet, the only solution is that $a=\tilde{a}=0$, i.e. all supersymmetries are broken. In the meantime there is no constraint on the abelian symmetries generated by $J$ and $\tilde{J}$. One can also limit the boundary to a subspace of the odd parts. If a condition is imposed among $\theta$, the left moving $N=2 \mathrm{SCA}$ will be broken down to a $N=1 \mathrm{SCA}$. Similarly one can do the same for the right movers. This returns us to boundary conditions of $N=1 \mathrm{SCA}$ solved in the last section. Here let us consider instead the condition

$$
\tilde{\theta}=\mathbf{k}(\sigma) \theta
$$

which can preserve a diagonal part of the two $N=2$ SCA's.

Requiring this equation to be invariant under (eq. 4.6) leads to

$$
\tilde{a}=\mathbf{k} \epsilon, \quad \tilde{\xi} \sigma^{3}=\xi \hat{\mathbf{k}} \sigma^{3} \hat{\mathbf{k}}^{-1}+\imath z^{-1} \epsilon \partial_{\sigma} \hat{\mathbf{k}} \hat{\mathbf{k}}^{-1}
$$

where $\hat{\mathbf{k}}=-\imath \exp (\imath \sigma) \mathbf{k}$. There are a number of constraints on $\hat{\mathbf{k}}$, a minimal set of which are

$$
\hat{\mathbf{k}}^{\top} \sigma^{1} \hat{\mathbf{k}}=\sigma^{1}, \quad \sigma^{3} \hat{\mathbf{k}} \sigma^{3}= \pm \hat{\mathbf{k}} .
$$

Corresponding to the choice of sign in the last equation, there are two families of solutions for $\hat{\mathbf{k}}$. It can either be $\exp \left(\alpha \sigma^{3}\right)$ or $\sigma^{1} \exp \left(\alpha \sigma^{3}\right)$ Correspondingly $\tilde{\gamma}=\gamma$ and $\tilde{\gamma}=-\gamma$. Hence the boundary types allowed depend on the $\gamma$ value of the superspace. $\lambda=\tilde{\lambda}=0$ is the critical value allowing all possibilities. On top of that, the boundary has to be compatible with the reality condition, which means

$$
\tilde{\mathrm{g}} \mathbf{k}=\mathbf{k}^{*} \mathrm{~g} .
$$

From this we infer that $\alpha$ is real and imaginary respectively for noncompact and compact $N=2$.

A reparameterization automorphism acts on the boundary conditions as

$$
\mathbf{k} \rightarrow \tilde{\mathbf{f}} \mathbf{k f}^{-1},
$$

which can be used to bring $\hat{\mathbf{k}}$ to 1 . However, as for the case of $N=1$, this does not mean there is only one type of boundary. Consider first the noncompact $N=2$. The solutions for $\mathbf{k}$ correspond to a map from the boundary to $O(1,1)$ and consists of four components:

$$
\hat{\mathbf{k}}= \pm e^{\phi \sigma^{3}}, \text { or } \pm \sigma^{1} e^{\phi \sigma^{3}} \text {. }
$$

To bring them to 1 one has to make use of the four components of the reparameterization automorphism for noncompact $N=2$. Consider a worldsheet with more than one boundaries. It is not hard to see that $\mathbf{k}$ can be brought to 1 on all boundaries if and only if on all 
of them it lies in the same class. Hence they really are four distinct types of boundaries. For open string theory, the condition for the chiral fields can be deduced from (eq. 4.25) and (eq. 4.26). The four possibilities are grouped into two types. The two boundary conditions of type $\mathrm{B}$ are $\mathrm{f}$

$$
T=\tilde{T}, \quad J=\tilde{J}, \quad G^{+}= \pm \tilde{G}^{+}, \quad G^{-}= \pm \tilde{G}^{-}
$$

and the two of type $\mathrm{A}$ are

$$
T=\tilde{T}, \quad J=\tilde{J}, \quad G^{+}= \pm \tilde{G}^{+}, \quad G^{-}= \pm \tilde{G}^{-} .
$$

Correspondingly there are four type of boundary state. They are distinguished by their set of annihilators. For type B they are

$$
\left(L_{m}-\tilde{L}_{-m}\right), \quad\left(J_{m}+\tilde{J}_{-m}\right), \quad\left(G_{m}^{+} \pm \imath \tilde{G}_{-m}^{+}\right), \quad\left(G_{m}^{-} \pm \imath \tilde{G}_{-m}^{-}\right)
$$

and for type A they are

$$
\left(L_{m}-\tilde{L}_{-m}\right),\left(J_{m}-\tilde{J}_{-m}\right),\left(G_{m}^{+} \pm \imath \tilde{G}_{-m}^{-}\right), \quad\left(G_{m}^{-} \pm \imath \tilde{G}_{-m}^{+}\right)
$$

For compact $N=2 \mathrm{SCA}$ the situation is more subtle. The solutions for $\hat{\mathbf{k}}$ are

$$
\hat{\mathbf{k}}=\exp ^{\left(\imath \alpha \sigma^{3}\right)} \text { or } \sigma^{1} \exp ^{\left(\imath \alpha \sigma^{3}\right)}
$$

It is a map from the boundary to $O(2)$. When the boundary is not a loop, say the time-like trajectory traced out by a propagating open string, there are two disjoint classes corresponding to the two components of $O(2)$, hence two type of boundaries. They are just the type A and $\mathrm{B}$ above. Because a shift $\alpha \rightarrow \alpha+\pi$ changes the sign of all odd elements, the subtypes within A or B are no longer distinct. Algebraically this is because the sign changing map is an outer automorphism for the noncompact $N=2$ but an inner one for the compact $N=2$. The two types are exactly what have been classified in 4 . 3

However, when the boundary is a loop, for example corresponding to a boundary state, there are two infinite classes of solutions parameterized by an integer $s$. They corresponding to a map from the boundary loop to the two components of $O(2)$ with winding number $s$. Therefore there are $2 \mathbb{Z}$ possible types of boundary states. While the boundary conditions for zero winding are just those of type $A$ (eq. 4.34) or $B$ (eq. 4.33) (with the subtypes no

\footnotetext{
${ }^{\ddagger}$ This nomenclature adheres to the convention in the literature $[$.

$\S$ Related boundary conditions were observed in the context of topological $\sigma$-models in an example of the A-type in 20] and considered for both types in [21].
} 
longer distinct), the infinite series corresponding nontrivial winding are not known before. A B-type boundary states $\left.\left|B_{s}\right\rangle\right)$ with winding number $s$ are annihilated by

$$
\begin{aligned}
& \left(J_{n}+\tilde{J}_{-n}+s \frac{c}{3} \delta_{n, 0}\right), \\
& \left(L_{n}-\tilde{L}_{-n}-s \tilde{J}_{-n}-s^{2} \frac{c}{6} \delta_{n, 0}\right), \text { and } \\
& \left(G_{n}^{ \pm}+\imath \tilde{G}_{-n \pm s}^{ \pm}\right) .
\end{aligned}
$$

An A-type boundary states $\left.\left|A_{s}\right\rangle\right)$ with winding number $s$ are annihilated by

$$
\begin{aligned}
& \left(J_{n}-\tilde{J}_{-n}+s \frac{c}{3} \delta_{n, 0}\right), \\
& \left(L_{n}-\tilde{L}_{-n}+s \tilde{J}_{-n}-s^{2} \frac{c}{6} \delta_{n, 0}\right), \text { and } \\
& \left(G_{n}^{ \pm}+\imath \tilde{G}_{-n \mp s}^{\mp}\right) .
\end{aligned}
$$

Everything in these equations except the terms involving the central charge are directly determined by substituting (eq. 4.25) and (eq. 4.26) in (eq. 4.4) and its right moving counterpart with $\hat{\mathbf{k}}=\exp \left(\imath s \sigma \sigma^{3}\right)$ for type $\mathrm{B}$ and $\hat{\mathbf{k}}=\sigma^{1} \exp \left(\imath s \sigma \sigma^{3}\right)$ for type A. The result is consistent with the $N=2$ algebra without central extension. The central terms are obtained by demanding consistency with the full algebra, in particular $\left\{G_{m}^{+}, G_{-m}^{-}\right\}$.

Now that these states are found, it is natural to look for their algebraic interpretation. The original type $\mathrm{A}$ and $\mathrm{B}$ boundary conditions were classified [4] by the $Z_{2}$ outer automorphism of $N=2 \mathrm{SCA}$ which inverts the sign of the $U(1)$ current:

$$
L \rightarrow L, \quad J \rightarrow-J, \quad G^{ \pm} \rightarrow G^{\mp}
$$

These two new series should also correspond to families of outer automorphism. In fact there is a well-known family of transformations of $N=2 \mathrm{SCA}$ parameterized by a real number $s$. It is known as spectral flow and changes the boundary condition of $G^{ \pm}$by $\exp (2 \pi \imath s)$ [22]:

$$
\begin{aligned}
J_{n} & \rightarrow J_{n}+s \frac{c}{3} \delta_{n, 0} \\
L_{n} & \rightarrow L_{n}+s J_{n}+s^{2} \frac{c}{6} \delta_{n, 0} \\
G_{n}^{ \pm} & \rightarrow G_{n \pm s}^{ \pm} .
\end{aligned}
$$

It was obtained by the adjoint action of an affine- $U(1)$ transformation with monodromy $\exp (2 \pi \imath s)$. The case of $s= \pm 1 / 2$ is important for understanding spacetime supersymmetry in $N=2$ compactifications of superstring. When $s$ is integral, it does not change the periodicity condition of $G^{ \pm}$so becomes an automorphism of the $N=2 \mathrm{SCA}$. It is in fact 
an outer automorphism because the affine- $U(1)$ transformation leading to it corresponds to an element of the loop group of $U(1)$ disconnected from the identity. It It is easy to see that the $B_{s}$ series of boundary states are related to this automorphism. Now, by following (eq. 4.39) with a conjugation automorphism, one obtains another outer automorphism:

$$
\begin{aligned}
G_{n}^{ \pm} & \rightarrow G_{n \mp s}^{\mp} \\
J_{n} & \rightarrow-J_{n}-s \frac{c}{3} \delta_{n, 0} \\
L_{n} & \rightarrow L_{n}+s J_{n}+s^{2} \frac{c}{6} \delta_{n, 0}
\end{aligned}
$$

which evidently corresponds to the $A_{s}$ series of boundary states.

This view of the nontriviality of the boundary conditions fit nicely with the above derivation of these boundary conditions from geometry of the superworldsheet. $s$ is the winding numbers of $\mathbf{k}$ in the loop group of $O(2)$. If one insists on unwinding it by say $\Xi$ without introduce winding at other boundary components, one has to introduce either a Chern class for the $U(1)$ bundle or, as a singular limit, the insertion of integral spectral flow operators. It is also worth noting that just as mirror symmetry relates type $\mathrm{A}$ and $\mathrm{B}$ boundary conditions, so it also relates $A_{s}$ and $B_{-s}$.

\section{$5 \quad$ Free field realization}

Here I give a free field realization of the two new infinite series of boundary states in (eq. 4.36) and (eq. 4.37), in terms of $d$ pairs of complex bosons and complex fermions with a Hermitian metric $\left(\mathcal{G}_{i \bar{j}}\right)^{*}=\mathcal{G}_{\bar{i} j} \equiv \mathcal{G}_{j \bar{i}}$ :

$$
\left[\alpha_{m}^{i}, \alpha_{n}^{\bar{j}}\right]=m \mathcal{G}^{i \bar{j}} \delta_{m+n}, \quad\left\{\psi_{m}^{i}, \psi_{n}^{\bar{j}}\right\}=\mathcal{G}^{i \bar{j}} \delta_{m+n}, \quad i, \bar{j}=1 \ldots d .
$$

and the same for the right movers. It is sufficient to consider the sector with half integral moding for all the fermions. The compact $N=2 \mathrm{SCA}$ is realized as (with normal ordering)

$$
\begin{aligned}
G_{n}^{+} & =\sum_{p+q=n} \psi_{p}^{i} \alpha_{q}^{\bar{i}}, & G_{n}^{-} & =\sum_{p+q=n} \psi_{p}^{\bar{i}} \alpha_{q}^{i}, \\
J_{n} & =\sum_{p+q=n} \psi_{p}^{i} \psi_{q}^{\bar{i}}, & L_{n} & =\sum_{p+q=n} \alpha_{p}^{i} \alpha_{q}^{\bar{i}}+\frac{q-p}{2} \psi_{p}^{i} \psi_{q}^{\bar{i}} .
\end{aligned}
$$

\footnotetext{
『Recently the related spectral flow in and nontrivial elements of the loop group of $S L(2, \mathbb{R})$ has found application in a different capacity [23].
} 
It is easy to verify that (eq. 4.36) is satisfied provided that |\rangle$) B_{s}$ is annihilated by

$$
\begin{array}{ll}
\left(\alpha_{m}^{i}+R_{j}^{i} \tilde{\alpha}_{-m}^{j}\right), & \left(\alpha_{m}^{\bar{i}}+R_{{ }_{j}}^{\bar{i}_{j}} \tilde{\alpha}_{-m}^{\bar{j}}\right), \\
\left(\psi_{m}^{i}+\imath R_{j}^{i}{ }_{j} \tilde{\psi}_{-m+s}^{j}\right), & \left(\psi_{m}^{\bar{i}}+\imath R^{\bar{i}} \bar{\psi}_{j} \tilde{\psi}_{-m-s}^{\bar{j}}\right),
\end{array}
$$

where $\left(R_{j}^{i}\right)^{*}=R_{\bar{j}}^{\bar{i}_{\bar{j}}}$ is unitary. Their kernel is $2^{|s|}$ dimensional. For $s \geq 0$, one solution can be constructed from the $S L(2, \mathbb{R}) \times S L(2, \mathbb{R})$ vacuum $|\emptyset\rangle\rangle$ as

$$
\begin{aligned}
\left.\left|B_{-s}\right\rangle\right)_{0}= & \exp \left[-\sum_{m>0} \frac{1}{m}\left(R_{i \bar{j}} a_{-m}^{i} \tilde{a}_{-m}^{\bar{j}}+R_{\overline{i j}} a_{-m}^{\bar{j}} \tilde{a}_{-m}^{i}\right)\right] \\
& \exp \left[-\sum_{m>0} \imath\left(R_{i \bar{j}} \psi_{-m}^{i} \tilde{\psi}_{-m-s}^{\bar{j}}+R_{\overline{i j}} \psi_{-m-s}^{\bar{i}} \tilde{\psi}_{-m}^{j}\right)\right] \\
& \left.\prod_{k>0}^{s}\left(\psi_{k-s}^{\bar{i}}+\imath R_{\bar{j}}^{\bar{i}_{j}} \tilde{\psi}_{-k}^{\bar{j}}\right)|\emptyset\rangle\right\rangle .
\end{aligned}
$$

Here $R_{\bar{i} j}=\mathcal{G}_{\bar{i} i} R_{j}^{i}$, and $R_{i \bar{j}}=\mathcal{G}_{i \bar{i}} R_{\bar{j}}^{\bar{i}}$. A complete set of basis can be formed by $\left.\left|B_{s}\right\rangle\right)_{0}$ and applying products of $\left(\psi_{k-s}^{\bar{i}}-\imath R_{\bar{j}}^{\bar{i}} \tilde{\psi}_{-k}^{\bar{j}}\right)$ on it for $0<k<s$.

For $s \leq 0$ one solution is

$$
\begin{aligned}
\left.\left|B_{s}\right\rangle\right)_{0}= & \exp \left[-\sum_{m>0} \frac{1}{m}\left(R_{i \bar{j}} a_{-m}^{i} \tilde{a}_{-m}^{\bar{j}}+R_{\overline{i j}} a_{-m}^{\bar{j}} \tilde{a}_{-m}^{i}\right)\right] \\
& \exp \left[-\imath \sum_{m>0}\left(R_{i \bar{j}} \psi_{-m+s}^{i} \tilde{\psi}_{-m}^{\bar{j}}+R_{\overline{i j}} \psi_{-m}^{\bar{i}} \tilde{\psi}_{-m+s}^{j}\right)\right] \\
& \left.\prod_{k>0}^{-s}\left(\psi_{-k}^{i}+\imath R_{j}^{i} \tilde{\psi}_{k+s}^{j}\right)|\emptyset\rangle\right\rangle .
\end{aligned}
$$

Applying $\left(\psi_{-k}^{i}-\imath R^{i}{ }_{j} \tilde{\psi}_{k+s}^{j}\right)$ on it for $0<k<-s$ and one can span the whole solution spaces.

$\left.\left|A_{s}\right\rangle\right)$ can be obtained similarly. The solitons lies in the common kernel of

$$
\begin{array}{cl}
\left(\alpha_{m}^{i}+R^{i}{ }_{j} \tilde{\alpha}_{-m}^{\bar{j}}\right), & \left(\alpha_{m}^{\bar{i}}+R_{j}^{\bar{i}} \tilde{\alpha}_{-m}^{j}\right), \\
\left(\psi_{m}^{i}+\imath R_{j}^{i} \tilde{\psi}_{-m-s}^{\bar{\psi}_{-m}}\right), & \left(\psi_{m}^{\bar{i}}+\imath R^{\bar{i}}{ }_{j} \tilde{\psi}_{-m+s}^{j}\right),
\end{array}
$$

which is again $2^{|s|}$ dimensional. Here $\left(R_{\bar{j}}^{i^{*}}\right)^{*}=R_{j}^{\bar{i}}$ is anti-unitary. For $s \geq 0$ one solution is

$$
\left.\left|A_{s}\right\rangle\right)_{0}=\exp \left[-\sum_{m>0} \frac{1}{m}\left(R_{i j} a_{-m}^{i} \tilde{a}_{-m}^{j}+R_{\overline{i j}} a_{-m}^{\bar{j}} \tilde{a}_{-m}^{\bar{i}}\right)\right]
$$

* In the language of D-branes, the $R$ matrix here for type $\mathrm{B}$ and later for type $\mathrm{A}$ encodes the geometric and gauge field configurations of a D-branes, as defined in [4]. It also has to satisfy certain consistency condition (unitary or anti-unitarity)as discussed ibid.

${ }^{\dagger}$ By that I mean $R^{i}{ }_{i} \mathcal{G}_{i \bar{j}} R^{\bar{j}}{ }_{j}=\mathcal{G}_{j \bar{i}}$. 


$$
\begin{aligned}
& \exp \left[-\sum_{m>0} \imath\left(R_{i j} \psi_{-m-s}^{i} \tilde{\psi}_{-m}^{j}+R_{\bar{i} \bar{j}} \psi_{-m}^{\bar{i}} \tilde{\psi}_{-m-s}^{\bar{j}}\right)\right] \\
& \left.\prod_{k>0}^{s}\left(\psi_{-k}^{i}+\imath R_{\bar{j}}^{i} \tilde{\psi}_{k-s}^{\bar{j}}\right)|\emptyset\rangle\right\rangle .
\end{aligned}
$$

Applying products of $\left(\psi_{-k}^{i}-\imath R^{i} \tilde{\psi}_{k-s}^{\bar{j}}\right)$ on $\left.\left|A_{s}\right\rangle\right)_{0}$, for $0<k<s$ to get a basis spanning the whole kernel.

For $s \leq 0$ one has

$$
\begin{aligned}
\left.\left|A_{s}\right\rangle\right)_{0}= & \exp \left[-\sum_{m>0} \frac{1}{m}\left(R_{i j} a_{-m}^{i} \tilde{a}_{-m}^{j}+R_{\overline{i j}} a_{-m}^{\bar{j}} \tilde{a}_{-m}^{\bar{i}}\right)\right] \\
& \exp \left[-\sum_{m>0} \imath\left(R_{i j} \psi_{-m}^{i} \tilde{\psi}_{-m+s}^{j}+R_{\overline{i j}} \psi_{-m+s}^{\bar{i}} \tilde{\psi}_{-m}^{\bar{j}}\right)\right] \\
& \left.\prod_{k>0}^{-s}\left(\psi_{k+s}^{\bar{i}}+\imath R^{\bar{i}}{ }_{j} \tilde{\psi}_{-k}^{j}\right)|\emptyset\rangle\right\rangle .
\end{aligned}
$$

Applying $\left(\psi_{k+s}^{\bar{i}}-\imath R^{\bar{i}} \tilde{\psi}_{-k}^{j}\right)$ on $\left.\left|A_{s}\right\rangle\right)_{0}$ for $0<k<-s$ to span the whole solution space.

It would be interesting to study these new types of boundary states in the context of general $N=2$ superconformal field theories. Another natural extension of the analysis in this paper would be to the cases of $N=3$ and $N=4$ SCA's.

I would like to thank V. Kac for illuminating replies to my question about outer automorphisms, L. Alvarez-Gaume, I. Brunner, M.B. Halpern, C. Helfgott, and Y. Oz for discussions.

\section{Appendix: Summary of $N=0,1,2$ SCA OPE's}

The Lie algebra of the mode operators of two chiral field $\phi$ and $\psi$ are determined by the singular part of their operator product expansion (sOPE), which is denoted in this paper by $\phi(z) \ldots \phi(w)$.

$N=0$

$$
T(z) \ldots T(w)=\frac{c / 2}{(z-w)^{4}}+\frac{T(w)}{(z-w)^{2}}+\frac{T(w)}{z-w} .
$$


$N=1$

$G$ is primary with conformal weight $3 / 2$. Its sOPE with itself is

$$
G(z) \ldots G(w)=\frac{2 c / 3}{(z-w)^{3}}+\frac{2 T(w)}{z-w} .
$$

$N=2$

$G^{ \pm}$and $J$ are primary with conformal weight $3 / 2$ and 1 . The rest of their sOPEs are

$$
\begin{aligned}
G^{+}(z) \ldots G^{+}(w) & =G^{-}(z) G^{-}(w)=0 \\
G^{+}(z) \ldots G^{-}(w) & =\frac{c / 3}{(z-w)^{3}}+\frac{J(w)}{(z-w)^{2}}+\frac{\partial J(w) / 2}{z-w}+\frac{T(w)}{z-w} \\
J(z) \ldots G^{ \pm}(w) & = \pm \frac{G^{ \pm}(w)}{z-w} \\
J(z) \ldots J(w) & =\frac{c / 3}{(z-w)^{2}} .
\end{aligned}
$$

\section{References}

[1] C. Albertsson, U. Lindstrom and M. Zabzine, $N=1$ supersymmetric sigma model with boundaries. I, (2001), hep-th/0111161.

[2] U. Lindstrom and M. Zabzine, $N=2$ boundary conditions for non-linear sigma models and Landau-Ginzburg models, (2002), hep-th/0209098.

[3] J. Callan, Curtis G., C. Lovelace, C. R. Nappi and S. A. Yost, Loop corrections to conformal invariance for type 1 superstrings, Phys. Lett. B206, 41 (1988).

[4] H. Ooguri, Y. Oz and Z. Yin, D-branes on Calabi-Yau spaces and their mirrors, Nucl. Phys. B477, 407-430 (1996), hep-th/9606112.

[5] J. Polchinski, Lectures on D-branes, (1996), hep-th/9611050.

[6] C. Albertsson, U. Lindstrom and M. Zabzine, $N=1$ supersymmetric sigma model with boundaries. II, (2002), hep-th/0202069.

[7] D. Fairlie and D. Martin, New lights on the Neveu-Schwarz model, Nuovo Cim. 18A, 373 (1973). 
[8] C. Montonen, Multiloop amplitudes in additive dual resonance models, Nuovo Cim. 19A, 69 (1974).

[9] P. S. Howe, Superspace and the spinning string, Phys. Lett. B70, 453 (1977).

[10] E. J. Martinec, Superspace geometry of fermionic strings, Phys. Rev. D28, 2604 (1983).

[11] D. Friedan, E. J. Martinec and S. H. Shenker, Conformal invariance, supersymmetry and string theory, Nucl. Phys. B271, 93 (1986).

[12] P. Di Vecchia, J. L. Petersen and H. B. Zheng, N=2 extended superconformal theories in two-dimensions, Phys. Lett. B162, 327 (1985).

[13] J. D. Cohn, N=2 superriemann surfaces, Nucl. Phys. B284, 349 (1987).

[14] E. B. Kiritsis, The structure of $n=2$ superconformally invariant 'minimal' theories: operator algebra and correlation functions, Phys. Rev. D36, 3048 (1987).

[15] K. Schoutens, $O(N)$ extended superconformal field theory in superspace, Nucl. Phys. B295, 634 (1988).

[16] J. L. Cardy, Boundary conditions, fusion rules and the verlinde formula, Nucl. Phys. B324, 581 (1989).

[17] Y.-K. E. Cheung, Y. Oz and Z. Yin, Families of $N=2$ strings, (2002), hep-th/0211147.

[18] To Appear .

[19] N. Ishibashi, The boundary and crosscap states in conformal field theories, Mod. Phys. Lett. A4, 251 (1989).

[20] P. Horava, Equivariant topological sigma models, Nucl. Phys. B418, 571-602 (1994), hep-th/9309124.

[21] E. Witten, Chern-Simons gauge theory as a string theory, Prog. Math. 133, 637-678 (1995), hep-th/9207094.

[22] A. Schwimmer and N. Seiberg, Comments on the $N=2, N=3, N=4$ superconformal algebras in two-dimensions, Phys. Lett. B184, 191 (1987).

[23] J. M. Maldacena and H. Ooguri, Strings in $A d S(3)$ and $S L(2, R) W Z W$ model. I, J. Math. Phys. 42, 2929-2960 (2001), hep-th/0001053. 